\title{
Spatial Distribution and Optimization Of Urban Surface Water Environment
}

\author{
Yifan Zhang ${ }^{1,}$ a, Haitao Jing 2 , b \\ ${ }^{1}$ Number 2001, ShiJi Road, GaoXin Zone, Jiaozuo City, Henan Province, China \\ ${ }^{2}$ Number 2001, ShiJi Road, GaoXin Zone, Jiaozuo City, Henan Province, China \\ a972370210@qq.com, bjht_6153@163.com
}

\begin{abstract}
Keywords: surface water environment; GIS; RS; spatial distribution; optimization
Abstract. The spatial distribution of urban surface water plays a significant role in the local environmental conditions, so it is important to optimize the spatial pattern of urban surface water environment. In recent year, with the rapid development of GIS and RS, its technologies have been used widely; GIS and RS have provided a new ideas and methods in the research on the spatial distribution and optimization of surface water. Based on Landsat- 8 remote sensing image classification results, firstly, the classification results of urban land features were obtained in ENVI, and then the spatial distribution of urban surface water environment was studied with the spatial statistical analysis of GIS. Finally, the purpose of this research is to optimize the local water environment. The result shows that the spatial distribution of surface water environment in target city is relatively reasonable but the northwestern and southern areas need much more optimization.
\end{abstract}

\section{Introduction}

Water is the source of life and plays a critical role in maintaining human survival and sustainable development of earth's ecological environment ${ }^{[1]}$. Water resources include surface water resources and Groundwater resources. It is no doubt that surface water resources occupy more important status in urban ecosystem. The spatial distribution and natural effect of surface water resources will affect more people and cause more problems in urban environment, comparing with other natural conditions ${ }^{[2]}$.

With the constantly development of GIS and RS, more and more researches began to apply them to various field. When it comes to the research on surface water resource, some researchers at domestic and abroad put forward their view in different aspects. At home, Xuwen Li processed satellite remote sensing images in Wuxi based on computer image processing and multivariate statistical methods, displaying the macroscopic appearance and spatial distribution characteristics of water quality in Wuxi ${ }^{[3]}$. Runke Wang proposed a decision based on the information from GIS and RS and achieved the dynamic management of water resources in Long Nan ${ }^{[4]}$. In University of Texas, C. D. McKinney developed Expert geographic information system for Texas water planning ${ }^{[5]}$.

Based on the remote sensing image in Landsat-8 and ENVI software, the images have been managed and get the classification image of spatial objects in research area; the of the spatial distribution of surface water environment in the studied city have been discussed, and in order to improve the ecosystem, this paper put forward a optimization suggestion in the spatial distribution of surface water.

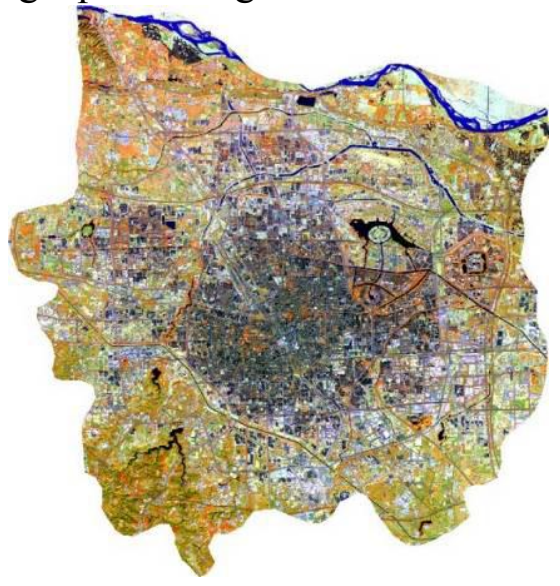

Fig 1 Remote sensing image

\section{Data Processing}

The image of this study is the remote sensing image of Landsat-8 in November 2017, coming from the Earth observation data sharing plan platform in Institute of Remote Sensing and Digital Earth, Chinese Academy of Sciences. Different band combination can expand the difference of 
feature bands to improve the readability of features. Performing the false color composite picture by band 5, 6, 4 aim to distinguish land and water. In this situation, the orange represent land and the blue represent water ${ }^{[6]}$. Then sharpen the image to improve the visual effects, compensate profile, highlight the edge information and make the image clearer. After that, subset the image by the city's administrative divisions of border is suitable for subsequent classification and analysis.

Table $1 \mathrm{~A}$ brief description of the band synthesis in Landsat-8 OLI

\begin{tabular}{cccc}
\hline $\mathbf{R}, \mathbf{G}, \mathbf{B}$ & Main function & $\mathbf{R , G}, \mathbf{B}$ & Main function \\
\hline $4,3,2$ & Naturally true color & $5,6,2$ & Healthy vegetation \\
$7,6,5$ & Penetrate the atmosphere & $7,6,4$ & City \\
$5,6,4$ & Land, water & $6,5,2$ & Agriculture \\
$5,4,3$ & Standard false color, vegetation & $7,5,4$ & Shortwave infrared \\
$7,5,3$ & Natural surface removing atmosphere & $6,5,4$ & Vegetation analysis \\
\hline
\end{tabular}

In this study, using the maximum likelihood in supervised classification to classify the image further. Supervised classification is a kind of common statistical classification with higher accuracy. It extracts all kinds of training patterns from the known types of training filed, and selects the characteristic variables, discriminant function and the discriminant rules, so that each pixel in the image is assigned to the classification of each given category ${ }^{[7]}$. Maximum Likelihood Classification assumes that each class of statistics in each band presents a normal distribution, and calculates the likelihood that a given pixel belongs to a certain training sample, so that the pixel is assigned to one with the highest likelihood among them ${ }^{[8}$. The discrimination rule is if the unclassified pixel $x$ satisfies Eq. $1, x \in w_{i}$.

$$
\mathrm{P}\left(w_{k}\right) P\left(\frac{x_{i}}{w_{k}}\right) \geq P\left(w_{l}\right) P\left(\frac{w_{i}}{w_{l}}\right), 1 \leq i \leq N, 1 \leq K, l \leq \mathrm{c}
$$

$N$ indicates the size of the image and c indicates the size of classification.

In order to extract the information about the spatial distribution of surface water within the city, the objects are divided into four categories by manual interpretation: wave, vegetation, bare land and buildings. The training patterns are drawn according to the four different types of ground objects. After the drawing is completed, the training patterns are optimized and refined to improve the classification accuracy. There still are some errors in the automatic classification of ENVI. At this time, the island pixels in the classification result and some patches may be deleted or combined by means of clump classes, sieve classes and the like. The classification result as shown in Fig. 2. Blue areas represent wave. Green areas represent vegetation. Orange areas represent buildings. Yellow areas represent bare land. The confusion matrix can be used to compare the classification of images and regions of interest to determine the accuracy of classification results. The overall accuracy is $95 \%$ and Kappa Coefficient is 0.92 in the results. It can be considered that the classification result has achieved a high degree of consistency.

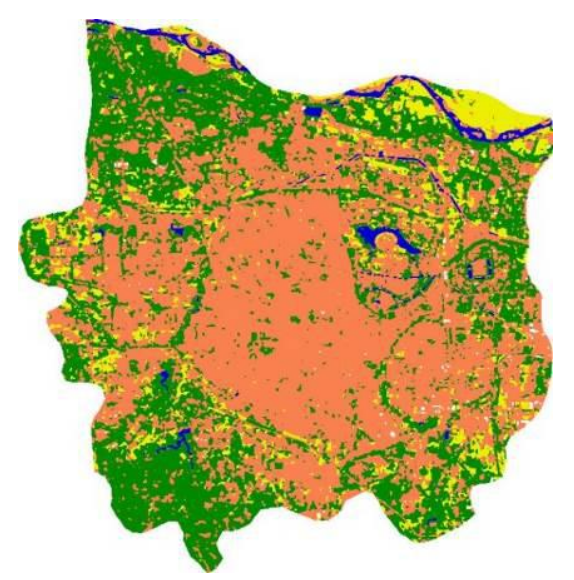

Fig. 2 Feature classification results

\section{Analysis}

The initial impression of the feature classification in study area by the classification results is not enough to rely on the classification results to get the relevant characteristics of urban surface water environment spatial distribution and must do further explore by the spatial analysis function of GIS. Strong spatial analysis function is the main feature of GIS, which is a spatial data analysis technology based on the location and morphological features of geographic objects, with the purpose of 
extracting and transmitting spatial information ${ }^{[9]}$. This study deeply analyzes the spatial distribution of urban surface water environment based on ArcGIS.

There are extremely rich content in GIS spatial analysis, including spatial query, spatial measurement, overlay analysis, buffer analysis, network analysis and spatial statistical classification analysis and so on ${ }^{[10]}$. The spatial statistical analysis is used to explore the spatial distribution of urban surface water. Spatial statistical analysis is the integration of spatial information into the classical statistical analysis to study the spatial relationship of things and phenomena related to the spatial location so as to reveal the spatial distribution of the elements. In the study of spatial distribution of urban surface water environment, using the directional distribution (standard deviation ellipse) in spatial statistics tools - measuring geographic distributions to measure the spatial distribution.

The formula for the standard deviation ellipse is given as Eq. 2:

$$
S D E_{x}=\sqrt{\frac{\sum_{i=1}^{n}\left(x_{i}-x\right)^{2}}{n}}, S D E_{y}=\sqrt{\frac{\sum_{i=1}^{n}\left(Y_{i}-P\right)^{2}}{n}} .
$$

Where $S D E_{x}$ and $S D E_{y}$ are the variance of Ellipse, $\bar{X}$ and $\bar{Y}$ represents the mean center for the features, $x_{i}$ and $y_{i}$ are the coordinates for feature $i^{[11]}$.

The semi major axis of the standard deviation ellipse indicates the direction of data distribution and the short axis indicates the range of data distribution ${ }^{[12]}$. From the distribution of the surface water environment in the study area, it is obvious that the distribution direction of the surface water environment in the studied cities is focused in northern parts and the distribution range is also concentrated in this area. In contrast, the spatial distribution of surface water in the northwestern and southern parts of the city is relatively poor, especially in the central and southern parts of the country, where there is almost no surface water. After superimposing building layers, it is found that the area with poorly distributed surface water environment is the densely distributed area of buildings. Due to the living environment and economic development, the distribution of surface water environment in these areas is unbalanced. Considering the needs of the ecological environment and the city's economic development, these areas should be given special attention in the future planning of urban surface water environment. The distribution of surface water in the study area is shown in Fig. 3.

\section{Optimization}

According to the results of the spatial statistical analysis in the previous chapter, it is clear that the spatial distribution of surface water environment in the northeast is more reasonable, while in the northwestern and southern regions is poorly distributed and requires further adjustment. In response to this situation, the following optimization suggestions are proposed for the surface water environment in the study area: Drainage of the Qili River in the central area to the south and construction of artificial reservoirs or lakes in the southeast and the south respectively will alleviate the poorly distributed spatial pattern

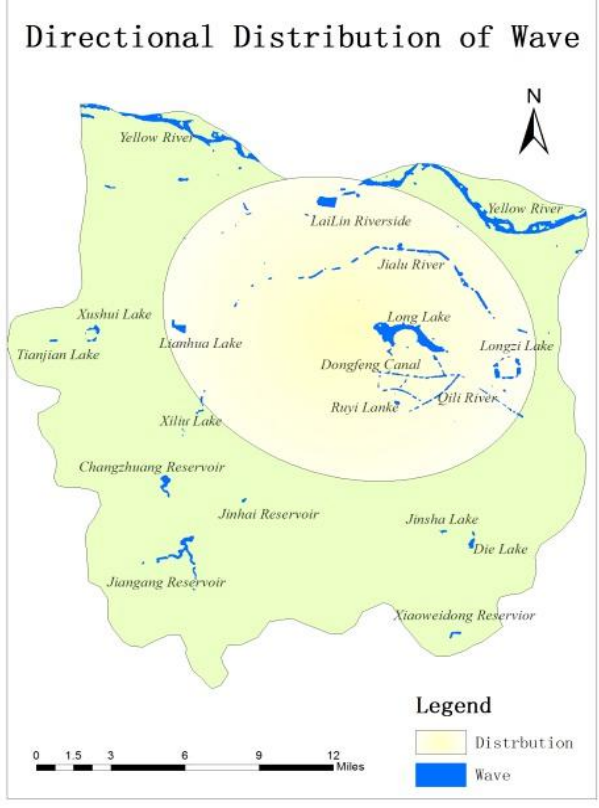

Fig. 3 Directional distribution of wave

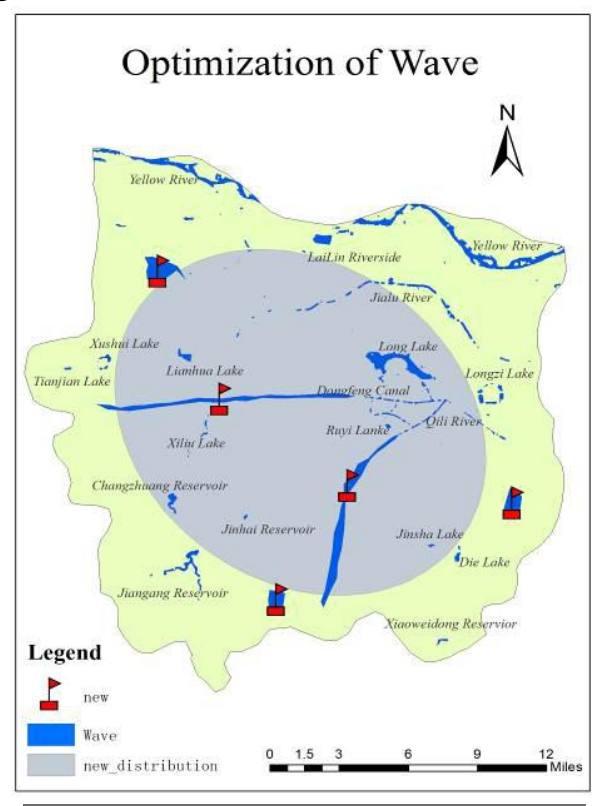

Fig. 4 Optimization of wave 
of the surface water environment in the south; Diverting the Dongfeng Canal located in the central area to the northwest and then building artificial reservoirs or lakes in the northwest so as to alleviate the poorly distributed surface water environment pattern in the northwest. The optimized area is distributed by the red flag on behalf of urban surface water environment spatial pattern. The optimized result is shown in Fig. 4.

\section{Conclusions}

The rise of GIS technology and remote sensing has provided new ideas and directions for the solution of related water conservancy issues. In addition to the research on the spatial distribution of urban surface water environment, it has also been applied to disaster prevention and control of floods, wading geological disasters monitoring and assessment, remote sensing estimation of soil moisture and evapotranspiration, groundwater monitoring, monitoring of water environment, ecological environment, soil and water conservation, etc. ${ }^{[13]}$. Except GIS and RS, the GPS in $3 \mathrm{~S}$ technology also can be used to accurately locate the measured data on the ground to further improve the management of the water environment to a higher degree of accuracy. In the future, when refers to the study of spatial distribution of surface water environment, the combination of $3 \mathrm{~S}$ technology will become an inevitable trend.

\section{Acknowledgements}

This work was supported by the Henan Province based frontier project, Henan Province "cartography" bilingual demonstration course project and Henan province "digital mapping and map synthesis" graduate quality course project.

\section{References}

[1] Y.Lv, in: Characteristics of urban water resources and existing problems, edited by Human Resources \& Social Sciences of Ability and Wisdom (2013)

[2] H.Wang, in: City Water Resource Management Facing Problems and Solutions, edited by Urbanism and architecture (2013)

[3] XW.Li in: Satellite Remote Sensing Analysis of Water Environment in Wuxi City, edited by volume 12 of Shanghai Environmental Sciences (1993)

[4] RK.Wang in: Application Research on Long nan Water Resources Management Based on GIS and RS, edited by volume 29 of Journal of Lanzhou University of Arts and Science (Natural Sciences)(2015)

[5] C D Mckinney in. Expert geographic information system for Texas water planning, edited by volume 22 of Journal of Water Resources Planning and Management (1993)

[6] LY.Niu, XY.Zhang, JY.Zheng, SD.Cao and HJ.Ruan in: Extraction of Cultivated Land Information in Shandong Province Based on Landsat8 OLI Data, edited by volume 34 of Chinese Agricultural Science Bulletin(2014)

[7] JF.Jia in: Compare of ENVI Remote Sensing Image Classification Methods, edited by Western Resources (2014)

[8] K.Sun and TD.LU in: Comparison of Supervised Classification Methods in Remote Sensing Image Classification edited by volume 35 of Jiangxi Science

[9] GA.Tang and X.Yang: Geographic Information System Spatial Analysis Experimental Course (Science Press, China 2015)

[10] XJ.Li in: Study of GIS- Based Spatial Analysis Methods, edited by Zhejiang University (2007)

[11] ESRI in: ArcGIS10.2 help

[12] Information on http://blog.csdn.net/allenlu2008/article/details/47780405]

[13] JR.Li in: Advance of hydrographic remote sensing with time edited by volume 47 of SHUILI XUEBAO (2016) 